OPEN ACCESS

Edited by:

Harinder Singh,

J. Craig Venter Institute, United States

Reviewed by:

Sipeng Shen,

Nanjing Medical University, China

Sudipto Saha,

Bose Institute, India

${ }^{*}$ Correspondence:

Xiangqian Guo

xqguo@henu.edu.cn

${ }^{\dagger}$ These authors have contributed equally to this work

Specialty section:

This article was submitted to Bioinformatics and Computational

Biology,

a section of the journal

Frontiers in Genetics

Received: 30 October 2019 Accepted: 03 April 2020

Published: 26 May 2020

Citation:

Yan Z, Wang Q, Lu Z, Sun X, Song P, Dang Y, Xie L, Zhang L, Li Y,

Zhu W, Xie T, Ma J, Zhang Y and Guo X (2020) OSluca: An Interactive Web Server to Evaluate Prognostic Biomarkers for Lung Cancer.

Front. Genet. 11:420

doi: 10.3389/fgene.2020.00420

\section{OSluca: An Interactive Web Server to Evaluate Prognostic Biomarkers for Lung Cancer}

\author{
Zhongyi Yan ${ }^{1 t}$, Qiang Wang ${ }^{1 t}$, Zhendong Lu't, Xiaoxiao Sun ${ }^{1}$, Pengfei Song ${ }^{1}$, \\ Yifang Dang ${ }^{1}$, Longxiang Xie ${ }^{1}$, Lu Zhang ${ }^{1}$, Yongqiang $\mathrm{Li}^{1}$, Wan Zhu' ${ }^{2}$, Tiantian Xie ${ }^{3}$, \\ Jing $\mathrm{Ma}^{3}$, Yijie Zhang $^{3}$ and Xiangqian Guo ${ }^{1 *}$
}

\begin{abstract}
'Department of Predictive Medicine, Institute of Biomedical Informatics, Cell Signal Transduction Laboratory, Bioinformatics Center, Henan Provincial Engineering Center for Tumor Molecular Medicine, School of Software, School of Basic Medical Sciences, Henan University, Kaifeng, China, ${ }^{2}$ Department of Anesthesia, Stanford University, Stanford, CA, United States,

${ }^{3}$ Department of Respiratory and Critical Care Medicine, Huaihe Hospital of Henan University, Kaifeng, China
\end{abstract}

Lung cancer is the principal cause of leading cancer-related incidence and mortality in the world. Various studies have excavated the potential prognostic biomarkers for cancer patients based on gene expression profiles. However, most of these reported biomarkers lack independent validation in multiple cohorts. Herein, we collected 35 datasets with long-term follow-up clinical information from TCGA (2 cohorts), GEO (32 cohorts), and Roepman study (1 cohort), and developed a web server named OSluca (Online consensus Survival for Lung Cancer) to assess the prognostic value of genes in lung cancer. The input of OSluca is an official gene symbol, and the output web page of OSluca displays the survival analysis summary with a forest plot and a survival table from Cox proportional regression in each cohort and combined cohorts. To test the performance of OSluca, 104 previously reported prognostic biomarkers in lung carcinoma were evaluated in OSluca. In conclusion, OSluca is a highly valuable and interactive prognostic web server for lung cancer. It can be accessed at http:// bioinfo.henu.edu.cn/LUCA/LUCAList.jsp.

Keywords: survival, lung cancer, biomarker, prognosis, OSluca

\section{INTRODUCTION}

Lung cancer (LUCA) is an aggressive disease with leading mortality and incidence in the world. Based on histology, there are two types of LUCA, including non-small cell lung cancer (NSCLC), which accounts for $80 \%$ of LUCA and small cell lung cancer (SCLC), which accounts for approximately 20\% of LUCA (Raponi et al., 2006; Bray et al., 2018). NSCLC can be further sub-divided into four subtypes, including adenocarcinoma, squamous cell carcinoma, large cell carcinoma, and bronchioloalveolar carcinoma (Ramalingam et al., 2011). Classical histological subtypes indeed play a dominant role in treatment and prognosis of lung cancer. Recently, reclassification of lung cancer based on tumor biomarkers improves lung cancer therapy (Beer et al., 2002; Hoadley et al., 2018).

Many studies have demonstrated that using clinical-association-prognostic biomarkers can assist the characterization of cancer subtypes and provide new insights of cancer recurrence and patients response to more precise therapies (Meyerson and Carbone, 2005; Bild et al., 2006; 
Raponi et al., 2006). It is worth noting that numerous single- or multi-prognostic biomarkers have been identified using highthroughput profiling methods (Raponi et al., 2006). By mining a mass of these profiling data deposited in public database, metaanalysis has exploited potential prognostic genes, such as KRT8 (Xie et al., 2019a). However, for biologists and clinicians, it is technically difficult to analyze these massive public data to screen and develop prognostic biomarkers. Previously, we have built several web servers of prognostic biomarker analysis for breast cancer, esophageal carcinoma, etc. (Wang et al., 2019a,b,c, 2020; Xie et al., 2019b,c; Yan et al., 2019; Zhang et al., 2019, 2020; Dong et al., 2020). In this current study, we have integrated bulky RNA expression profiles of lung cancer with clinical survival information, mainly from TCGA (The Cancer Genome Atlas) and GEO (the Gene Expression Omnibus) databases, and built a prognostic analysis web server named OSluca (Online consensus Survival for Lung Cancer) to analyze and evaluate prognostic potency of gene in 35 independent lung cancer cohorts.

\section{MATERIALS AND METHODS}

\section{Collection of Lung Cancer Datasets}

The lung cancer cohorts for OSluca with expression profiling and clinical follow-up data were collected from PubMed, TCGA, ${ }^{1}$ and $\mathrm{GEO}^{2}$ by searching the keywords: "lung" AND "cancer" AND "survival" (Table 1). The dataset for each cohort that met these following criteria will be included in OSluca: (1) have RNA sequencing or gene microarray data; (2) have complete followup data, such as overall survival and status (Liu et al., 2018); (3) all the data were specific for lung cancer, not from secondary or metastatic lung tumor from other types of tumors; (4) the cohort size is no less than 30 cases. The primary clinical pathological characteristics of lung cancer patients are listed in Table $\mathbf{1 .}$

\section{Construction of OSluca Web Server}

Online consensus Survival for Lung Cancer is built in a tomcat server as previously described with minor modifications (Wang et al., 2019b,c; Xie et al., 2019b,c; Yan et al., 2019; Zhang et al., 2019). Briefly, front-end application was used for inputting query and displaying the results. Java and $\mathrm{R}$ package were used to analyze request and output the results. In addition, profiles and clinical information were stored in the SQL Server database. The prognostic significance of inputted gene is determined by analyzing the association of gene expression and survival time using the R package "survival." In addition, a genome-wide precalculation of Cox proportional regression for all the human genes were performed as well, and the home page of OSluca could display the survival analysis summary with a forest plot and a table of Cox proportional regression result for inputted gene in all cohorts with $P$-value and HR [ $(95 \%$ confidence interval (CI)] with the built-in upper $25 \%$ cutoff. The R package "forestplot" was used to produce the forest plot for inputted gene in OSluca web server.

${ }^{1}$ https://cancergenome.nih.gov/

${ }^{2}$ www.ncbi.nlm.nih.gov/geo/

\section{Validation of Previously Reported Prognostic Biomarkers of Lung Cancer in OSluca}

Keywords including "lung cancer," "survival," "biomarker," and "prognosis" were used to search biomarkers of lung cancer in NCBI PubMed. We finally obtained 104 prognostic biomarkers using the following criteria (Table 2): (1) immunohistochemistry (IHC) or qRT-PCR (qPCR) detection of biomarkers in primary cancer tissue; (2) a significant association between biomarker and survival; (3) the sample size must be above 50 cases; (4) the study was published in the English for full access.

\section{Statistical Analysis}

The association of lung cancer clinical factors and survival outcomes was analyzed by GraphPad Prism 8.0 software. The Cox proportional hazards regression and Kaplan Meier plot functions from R package "survival" were used in the OSluca to determine the association between gene expression and survival. The $P \leq 0.05$ was considered statistically significant.

\section{RESULTS}

\section{Clinical Characteristics of Lung Cancer Patients in OSluca}

To develop an online survival web server for lung cancer, we collected 35 published high-throughput profiling datasets of lung cancer with long-term follow-up information (2 TCGA datasets, 32 GEO datasets, and 1 Roepman dataset). TCGA comprises 513 lung adenocarcinoma cases and 499 squamous cell carcinoma cases (Tables 1, 2). GEO cohorts and Roepman cohort had more than 4,000 samples and 172 samples, respectively, as shown in Table 2. 4,901 patients have OS (overall survival) data; 2,176 patients have DSS (disease-specific survival) data; and 2,075 patients have PFI (progression-free interval or recurrencefree survival) data, while 608 patients have DFI (disease-free interval) data. The results showed that the patients with lung adenocarcinoma significantly survive longer than those of other histological lung cancer, and small cell lung cancer is associated with the worst prognosis compared to other types of lung cancer (Figure 1A). Moreover, other clinical characteristics can also prominently affect patients' prognosis, such as gender $(P<0.0001)$, stage $(P<0.0001)$, p-TNM stage $(P<0.0001)$, and smoking status $(P<0.0001)$ (Figures 1B-E). Besides, these risk factors can influence other survival endpoints, such as PFI (data not shown). These results are in accordance with previous researches (Mao et al., 2016; Bray et al., 2018).

\section{Construction and Usage of Prognostic Web Server OSluca}

Online consensus Survival for Lung Cancer includes a set of optional clinico-pathological factors, such as age, sex, histological type, grade, smoking status, and so on. Four survival endpoints can be selected basing on original patient outcomes, containing OS, DSS, DFI, and PFI (Liu et al., 2018). In order to make the 
TABLE 1 | Summary of clinical characteristics of lung cancer cohorts in Online Consensus Survival for Lung Cancer (OSluca).

\begin{tabular}{|c|c|c|c|c|c|c|c|}
\hline & \multicolumn{5}{|c|}{ NSCLC } & \multirow[t]{2}{*}{$\operatorname{SCLC}(N=223)$} & \multirow[t]{2}{*}{ \#NA $(N=85)$} \\
\hline & NSCLC, Total $(N=4937)$ & $A D(N=3345)$ & $\operatorname{scc}(N=1381)$ & $\operatorname{LCC}(N=197)$ & NOS $(N=194)$ & & \\
\hline Age, year & $64(13-91)$ & 64 (13-90) & 66 (39-83) & 63 (39-81) & $62(22-80)$ & 64 (40-83) & $58(15-82)$ \\
\hline \multicolumn{8}{|l|}{ Gender } \\
\hline Male, \% & 52.6 & 46.9 & 68.3 & 77.2 & 12.9 & 58.1 & 50 \\
\hline Female, \% & 38.8 & 47.7 & 23.7 & 18.1 & 12.4 & 41.9 & 50 \\
\hline \#NA, \% & 8.6 & 5.4 & 8.0 & 4.7 & 73.7 & 0 & 0 \\
\hline \multicolumn{8}{|l|}{ Stage ${ }^{\star}$} \\
\hline I, $n$ & 2301 & 1,653 & 567 & 66 & 28 & 10 & 9 \\
\hline$\|, n$ & 889 & 500 & 347 & 27 & 15 & 5 & 4 \\
\hline III, $n$ & 595 & 366 & 199 & 18 & 12 & 2 & 3 \\
\hline $\mathrm{IV}, n$ & 101 & 73 & 13 & 2 & 13 & 0 & 0 \\
\hline $\begin{array}{l}\text { T stage } \\
\text { 1/2/3/4/\#NA }\end{array}$ & 646/1074/230/103/2884 & 468/663/102/49/2063 & 155/362/109/39/716 & 20/44/17/9/107 & $3 / 5 / 2 / 6 / 178$ & $11 / 13 / 5 / 4 / 190$ & $28 / 20 / 10 / 6 / 21$ \\
\hline $\begin{array}{l}\text { N Stage } \\
0 / 1 / 2 / 3 / \# N A\end{array}$ & $1638 / 495 / 280 / 21$ & 1038/254/198/5/1859 & $549 / 218 / 70 / 7 / 537$ & 48/20/17/5/107 & $3 / 3 / 4 / 4 / 180$ & $14 / 4 / 12 / 6 / 187$ & $33 / 25 / 5 / 1 / 21$ \\
\hline M stage 0/1/\#NA & $1685 / 42 / 3210$ & $853 / 26 / 2466$ & $740 / 8 / 633$ & $82 / 2 / 113$ & 10/6/178 & $33 / 4 / 186$ & $63 / 2 / 20$ \\
\hline $\begin{array}{l}\text { Smoking/non- } \\
\text { smoking/\#NA }\end{array}$ & 1839/262/2836 & $1112 / 256 / 1977$ & $618 / 3 / 760$ & $40 / 1 / 156$ & 9/2/183 & $18 / 1 / 204$ & $9 / 8 / 68$ \\
\hline OS, mo & 46 (0.03-256) & 48 (0.03-242) & 41 (0.03-256) & $46(0.1-216)$ & 38 (0.5-208) & $51(2-211)$ & $68(2-244)$ \\
\hline DSS, mo & $42(0.03-256)$ & $43(0.19-242)$ & $41(0.03-256)$ & $45(1-216)$ & $36(6-76)$ & $24(2-140)$ & 69 (2-244) \\
\hline DFI, mo & $33(0.16-242)$ & $32(0.6-242)$ & $34(0.16-159)$ & - & - & - & - \\
\hline PFI, mo & 33 (0.03-242) & $36(0.03-242)$ & $30(0.03-180)$ & $53(1.8-164)$ & $4(0.23-54)$ & - & $30(2-73)$ \\
\hline
\end{tabular}

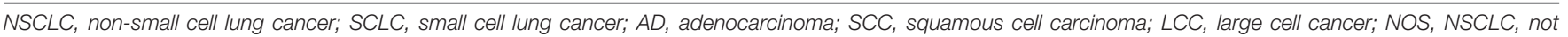

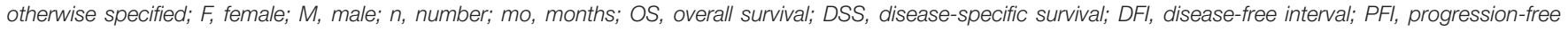
interval or recurrence free survival. *The stage only counts stages of lung cancer patients described in the original datasets; \#NA, data lost or unknown.

user clearly see the prognostic effect of interested gene, a metaanalysis is to summarize the prognostic value for each gene on the home page of OSluca. Briefly, after the user types the official gene symbol into the input box on the home page, OSluca will display the survival analysis summary with a forest plot and a table from Cox proportional regression in each cohort and combined cohorts (combining all the datasets together). Take the tumor suppressor gene TP53 (tumor protein p53) as an example and type "TP53" into the gene symbol box and click on "Survival analysis" (Figure 2A, left). The meta-analysis results with a forest plot and a survival table for the TP53 gene, will display the $P$-value and HR with 95\% CI of each cohort and the combined cohorts (Figure 2A, right). Then, the user can easily obtain KM plots of separate cohorts such as GSE30219 dataset by clicking on the "Go" button in the survival table (Figure 2B). In addition, it is also available to use a subgroup of certain cohort to obtain specific prognostic information with selectable risk factors, such as cutoff value, histological type, grade, etc. Briefly, OSluca can output survival rates displaying a forest plot and a survival table with KM plot and $P$-value to measure the association between the investigated gene and survival rate.

\section{Validation of Previously Reported Lung Cancer Prognostic Biomarkers in OSluca}

A search for lung cancer biomarkers was performed using a set of keywords in NCBI PubMed, including "lung cancer," "survival," "biomarker," and "prognosis." In total, we collected 104 published lung cancer prognostic biomarkers verified by IHC or qPCR
(Supplementary Table S1) to evaluate the performance of OSluca. For example, Hsu et al. reported that ERO1L (ERO1-like protein alpha, also named ERO1A) is significantly overexpressed in tumor tissue and could be as a poor prognostic biomarker for lung adenocarcinoma (Hsu et al., 2016). The prognostic analysis of ERO1L in OSluca showed that high expression of ERO1L gene is significantly associated with poor outcome in eight out of nine cohorts (Top 9 cohorts, the sample size above 150 cases) (Figures 3A-H), except the Roepman dataset (Figure 3I). Next, each published biomarker was investigated in the Top 9 cohorts in OSluca, and the results showed that approximately $66 \%$ of biomarkers (69/104) were consistent with original published findings (Supplementary Table S1). Meanwhile, OSluca can be used to perform the outcome metaanalysis of the interested gene that showed that 14\% (14/104) (Supplementary Table S1) of published prognostic genes have the similar prognostic values in one or multiple OSluca cohorts as reported in the literature, but these genes also showed the opposite outcomes in some other cohorts from OSluca. These genes need further investigations, such as the DDIT3 gene (Supplementary Figure S2 and Supplementary Table S1). In contrast, there are some prognostic biomarkers, which have been shown different outcomes between OSluca and previous findings. A total of $9 \%$ of the published prognostic genes showed opposite outcome results between OSluca and literatures (9/104) (see Supplementary Table S1), suggesting that these genes need further validation. For example, the transcription factor KLF15 (Krüppel-like factor 15) had been proven to be higher in tumor tissue than that of adjacent non-tumor tissue and played 
TABLE 2 | Clinico-pathological traits of lung cancer cohorts.

\begin{tabular}{|c|c|c|c|c|c|c|}
\hline Datasets & Cohorts & Platform & Histological type & Survival & Samples & References \\
\hline Rockville & GSE102287 & GPL570 & AD/SCC/NOS & OS & 32 & Mitchell et al., 2017 \\
\hline Heidelberg & GSE10245 & GPL570 & AD/SCC & OS & 58 & Kuner et al., 2009 \\
\hline Koto-ku & GSE1037 & GPL962 & AD/SCC/SCLC & OS & 61 & Jones et al., 2004 \\
\hline Basel & GSE11117 & GPL6650 & AD/SCC/NOS & OS & 41 & Baty et al., 2010 \\
\hline Nagoya & GSE11969 & GPL7015 & AD/SCC/LCC & os & 149 & Takeuchi et al., 2006 \\
\hline Groningen & GSE12428 & GPL1708 & SCC & OS & 34 & Boelens et al., 2009 \\
\hline Nagoya & GSE13213 & GPL6480 & AD & OS & 117 & Tomida et al., 2009 \\
\hline Toronto & GSE14814 & GPL96 & AD/SCC /NOS & OS/DSS & 133 & Zhu et al., 2010 \\
\hline Chapel Hill & GSE17710 & GPL9053 & SCC & OS/PFI & 56 & Wilkerson et al., 2010 \\
\hline Rotterdam & GSE19188 & GPL570 & AD/SCC/LCC & OS & 82 & Hou et al., 2010 \\
\hline Chapel Hill & GSE26939 & GPL9053 & $A D$ & os & 116 & Wilkerson et al., 2012 \\
\hline Dallas & GSE29013 & GPL570 & AD/SCC & OS/PFI & 55 & Xie et al., 2011 \\
\hline Lund & GSE29066 & GPL6947 & AD/SCC/SCLC & OS & 68 & Staaf et al., 2012, 2013 \\
\hline La Tronche & GSE30219 & GPL570 & AD/SCC/SCLC/LCC & OS/DFS & 293 & Rousseaux et al., 2013 \\
\hline Chuo-ku & GSE31210 & GPL570 & $A D$ & OS /PFI & 226 & Okayama et al., 2012 \\
\hline Durham & GSE3141 & GPL570 & AD/SCC & OS & 111 & Bild et al., 2006 \\
\hline Dallas & GSE31908 & GPL96/97 & $A D$ & OS & 30 & NA \\
\hline Houston & GSE33072 & GPL6244 & AD/SCC & $\mathrm{PFI}$ & 66 & Byers et al., 2013 \\
\hline Uppsala & GSE37745 & GPL570 & AD/SCC/LCC & $\mathrm{PFI}$ & 196 & Botling et al., 2013 \\
\hline Dallas & GSE41271 & GPL6884 & AD/SCC/LCC & OS/PFI & 275 & Sato et al., 2013 \\
\hline San Diego & GSE4573 & GPL96 & SCC & OS & 130 & Raponi et al., 2006 \\
\hline Nagoya & GSE4716 & GPL3696/3694 & AD/SCC/LCC & os & 50 & Tomida et al., 2004 \\
\hline Toronto & GSE50081 & GPL570 & AD/SCC/LCC & OS/DFS & 181 & Der et al., 2014 \\
\hline Brisbane & GSE5123 & GPL3877 & SCC & OS & 51 & Larsen et al., 2007b \\
\hline Brisbane & GSE5828 & GPL3877 & SCC & OS & 59 & Larsen et al., 2007a \\
\hline Brisbane & GSE5843 & GPL3877 & $A D$ & OS & 48 & Larsen et al., 2007c \\
\hline St. Louis & GSE6253 & GPL8300 & AD/SCC/NOS & DSS & 34 & Lu et al., 2006 \\
\hline Bethesda & GSE63459 & GPL6883 & $A D$ & OS & 33 & Robles et al., 2015 \\
\hline Stanford & GSE67639 & GPL570 & AD/SCC/NOS & OS & 1106 & Gentles et al., 2015 \\
\hline Rockville & GSE68465 & GPL96 & $A D$ & OS/PFI & $442 / 363$ & Shedden et al., 2008 \\
\hline Rockville & GSE68571 & GPL80 & $A D$ & OS & 86 & Beer et al., 2002 \\
\hline Seoul & GSE8894 & GPL570 & AD/SCC & $\mathrm{PFI}$ & 138 & Lee et al., 2008 \\
\hline $\mathrm{NIH}$ and NHGRI & TCGA & DCC & $A D$ & OS/DSS/DFI/PFI & $513 / 478 / 306 / 513$ & $\begin{array}{l}\text { The Cancer Genome } \\
\text { Atlas Research Network, } \\
\text { 2014; Liu et al., } 2018\end{array}$ \\
\hline $\mathrm{NIH}$ and NHGRI & TCGA & DCC & SCC & OS/DSS/DFI/PFI & 498/452/303/499 & $\begin{array}{l}\text { Hammerman et al., 2012; } \\
\text { The Cancer Genome } \\
\text { Atlas Research Network, } \\
\text { 2012; Liu et al., } 2018\end{array}$ \\
\hline Reopman & Roepman & & AD/SCC/LCC/NOS & OS & 172 & Roepman et al., 2009 \\
\hline
\end{tabular}

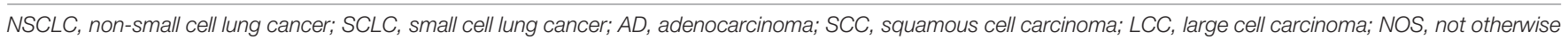
specified; OS, overall survival; DSS, disease-specific survival; DFI, disease-free interval; PFI, progression-free interval.

an important role in promoting proliferation and carcinoma diversification in lung adenocarcinoma, associated with poor prognostic outcome (Gao et al., 2017). It was not anticipated that the patients with high expression of KLF15 have better survival than those with low expression (Supplementary Table S1 and Supplementary Figure S1). The OSluca result for the KLF15 gene was consistent with other prognostic analysis tools (Gyõrffy et al., 2013; Anaya, 2016), such as the KM plotter $[P<0.001$, $\operatorname{HR}(95 \% \mathrm{CI})=0.4(0.28-0.58)]$. In addition, the remaining 12 of 104 previously published prognostic biomarkers (11\%) were not significant for prognostic analysis in the Top 9 cohorts in OSluca, but 8 of them $(8 / 12)$ are significant in one or multiple datasets other than the Top 9 cohorts in OSluca (data not shown). All in all, the OSluca server is an interactive and free web server for researchers to develop potential prognostic biomarkers for lung cancer.

\section{DISCUSSION}

Owing to tumor molecular heterogeneity, the prognosis of lung cancer patients is variable and difficult to predict. The prognosis of patients suffering from lung cancer had been demonstrated to be highly dependent on clinical factors 
A

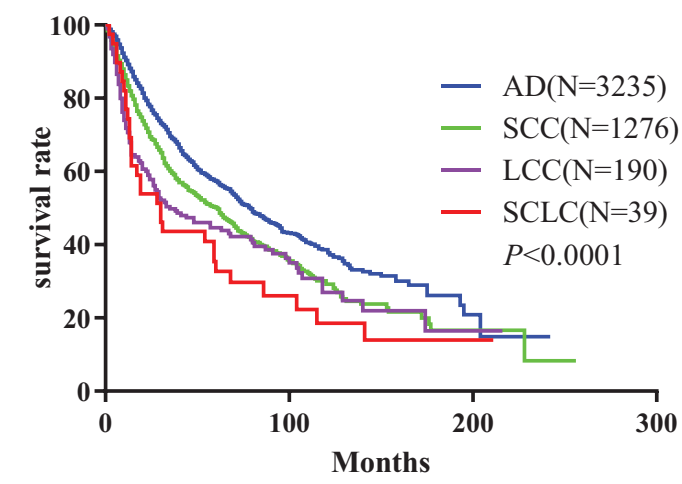

C

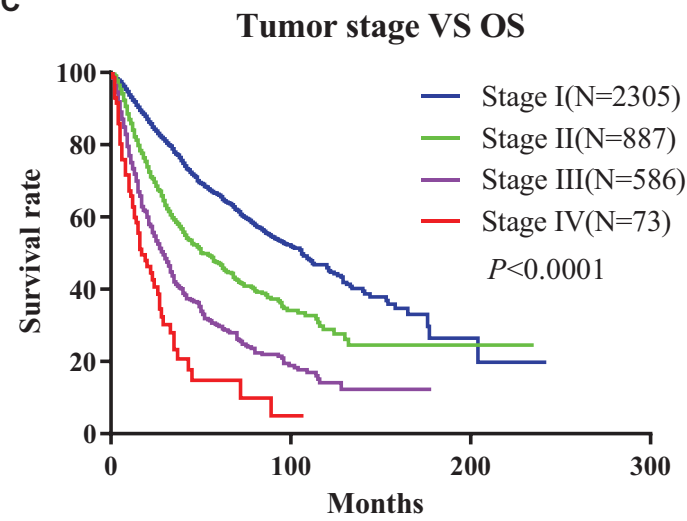

E
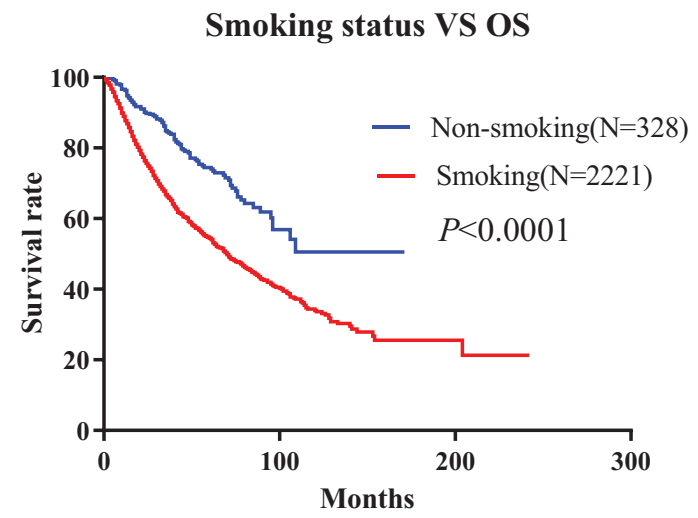

B

\section{Gender VS OS}

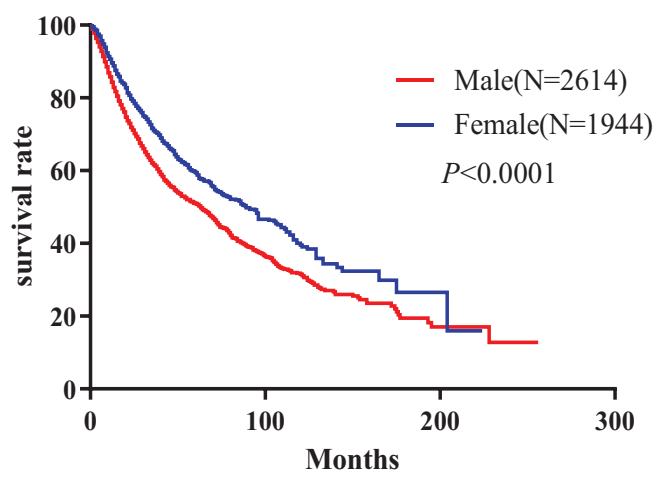

D p-TNM stage VS OS

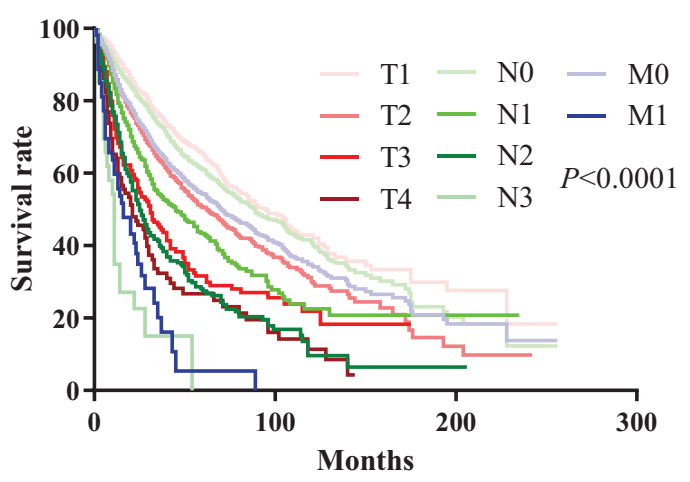

FIGURE 1 | Correlation between the clinico-pathologic characteristics and overall survival of lung cancer in Online Consensus Survival for Lung Cancer (OSluca). (A) Correlation between histological types and OS. (B) Correlation between gender and OS. (C) Correlation between tumor stages and OS. (D) Correlation between p-TNM stages and OS. (E) Correlation between smoking status and OS. OS, overall survival; AD, adenocarcinoma; SCC, squamous cell carcinoma; LCC, large cell cancer.

of the patient, such as histological type, smoking status, and so on. However, it is also an imperative need to exploit novel prognostic biomarkers for determining the risk of cancerous lesions and predicting lung cancer patient outcomes by all available means, especially by high-throughput sequencing technologies. However, one major challenge to non-bioinformatics researchers is how to integrate the highdimension profiling datasets of lung cancer and discover new biomarkers to potentially guide prognostic stratification. Previous studies had revealed that the online prognostic web 


\section{A}

\section{OSluca}

OSluca ( Online consensus Survival for Lunng Cancer) encompasses 35 expression datasets, provides 4 types of survival terms for 5741 patients of lung cancer.

Gene symbol: TP5

Survival analysis

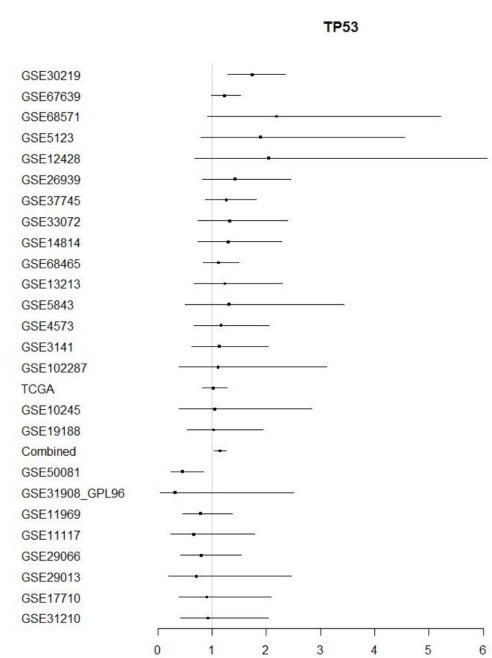

$\mathrm{HR}(95 \% \mathrm{Cl})$ $1.7437(1.2887-2.3594)$ $12262(0.9897 \sim 15191)$ $21888(0.9171 \sim 52238$ $1.8917(0.7859-4.5534)$ $2.0419(0.6859-6.0787)$ $1.426(0.8272 \sim 2.4583)$ $1.2631(0.8775-1.8182)$ $1.3289(0.7372-23955)$ $1.3007(0.7413-2.2823)$ $1.1167(0.8322 \sim 1.4984)$ $1.235(0.664-2.297)$ $13154(0.5035 \sim 34369)$ $1.1645(0.6622-2.0478)$ $1.132(0.6288 \sim 2038)$ $1.1093(0.3952 \sim 31136$ $1.0189(0.8163-1.2719)$ $1.0477(0.3863-2.8418$ $1.0278(0.545-1.9382$ $1.1482(1.0442-1.2626)$ $0.4491(0.2366 \sim 0.8524)$ $0.3134(0.0392 \sim 2.5081)$ $0.7908(0.4542-1.3769)$ $0.662(0.245-1.7885)$ $0.8045(0.4187-1.546)$ $0.7103(0.2041-2.4716)$ $0.904(0.3901-2.0946)$ $0.9229(0.4186 \sim 20348)$

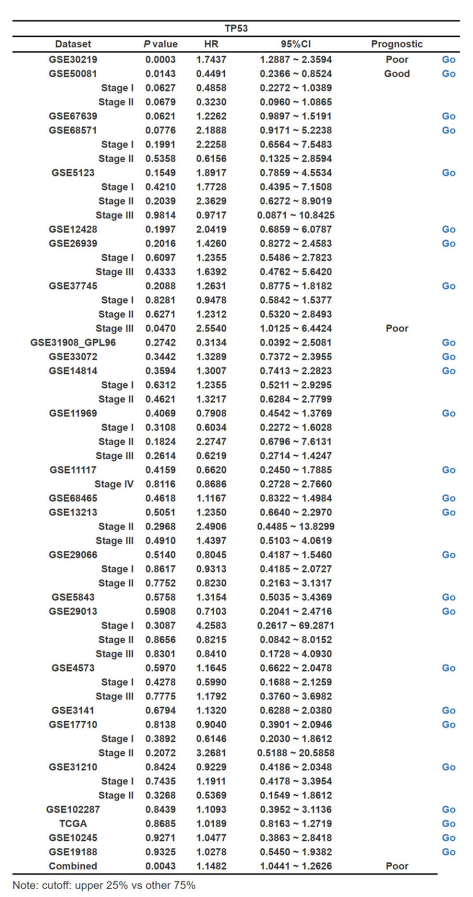

B

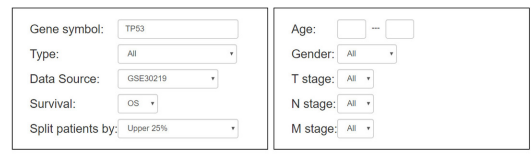

Kapian-Meier piot

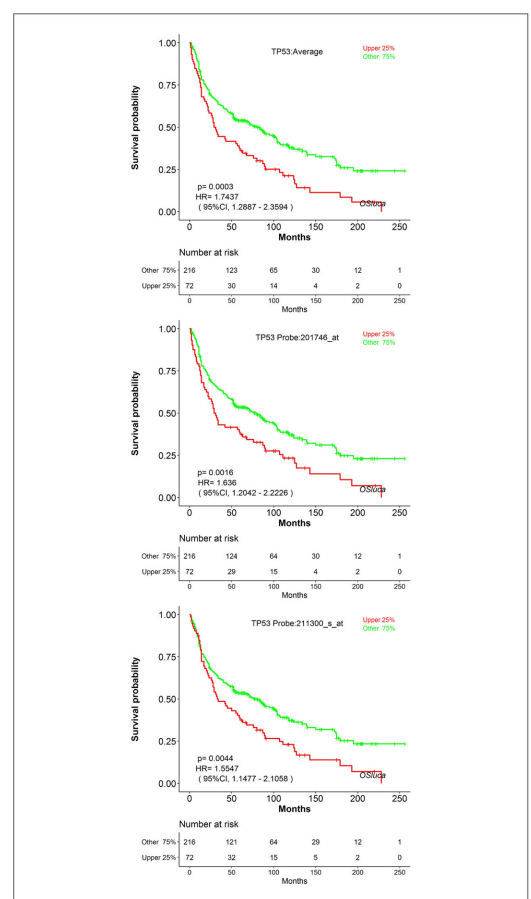

FIGURE 2 | The output home page and KM output web subpage in OSluca for lung cancer. (A) Home page of OSluca with TP53 gene survival analysis, containing prognostic meta-analysis of a forest plot and a survival table. (B) KM plots of TP53 gene in the GSE30219 cohort. Note: the cutoff value is the upper $25 \%$ vs. other $75 \%$. The "Combined" in forest plot and survival table means the overall prognostic significance of inputted gene in a pooling cohort with all the datasets. TP53, tumor protein $\mathrm{p} 53$. 

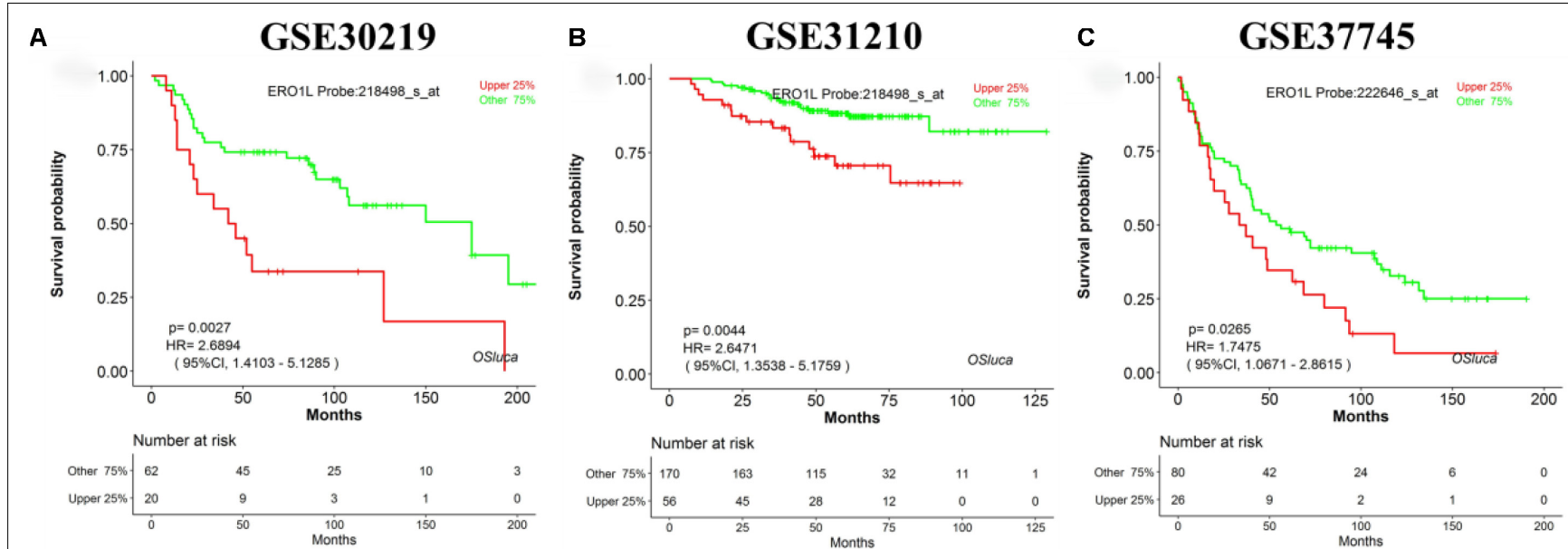

D
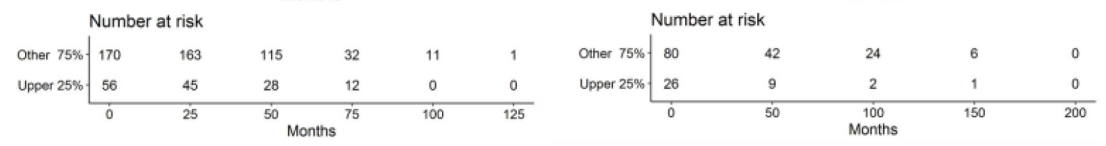

E

GSE50081

F

GSE67639
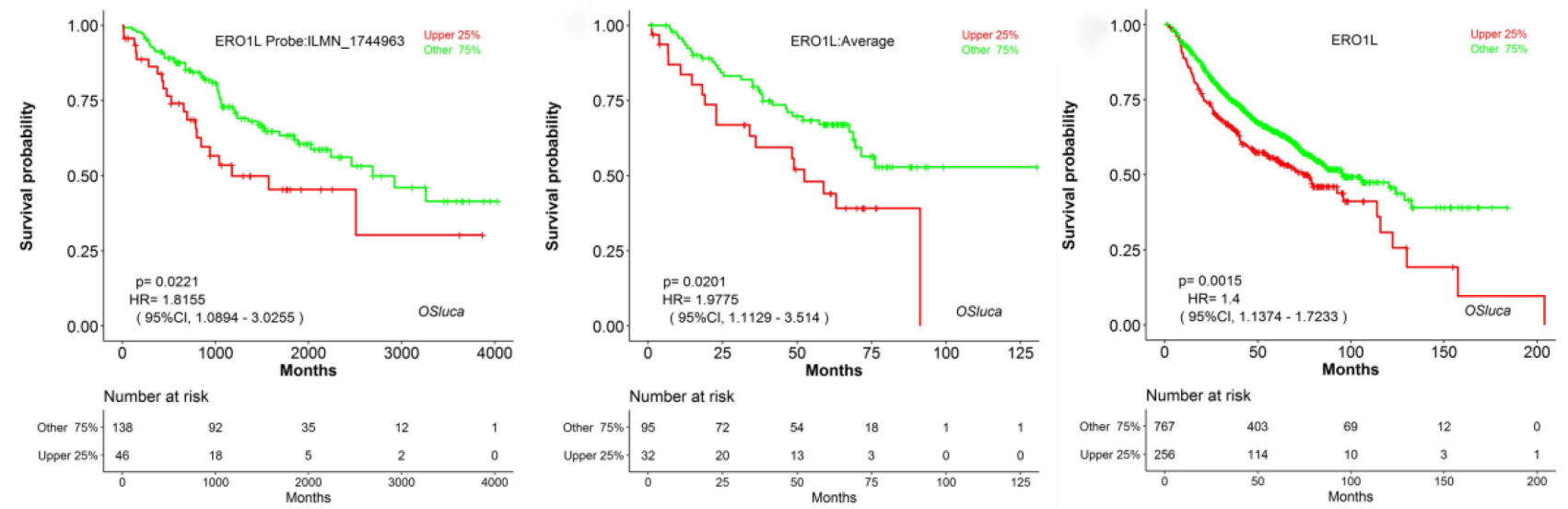

G

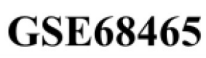

H

TCGA
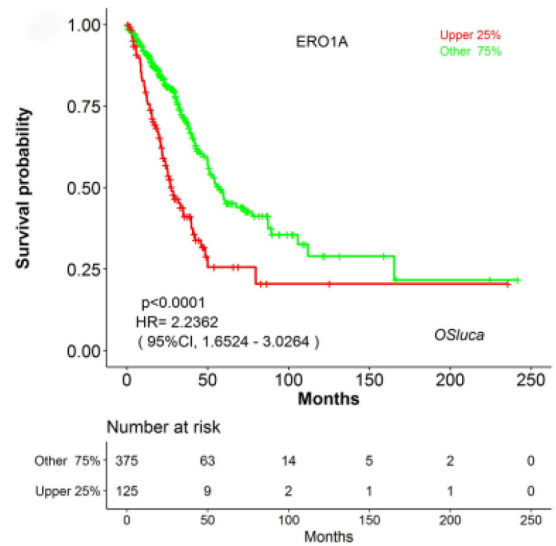

I
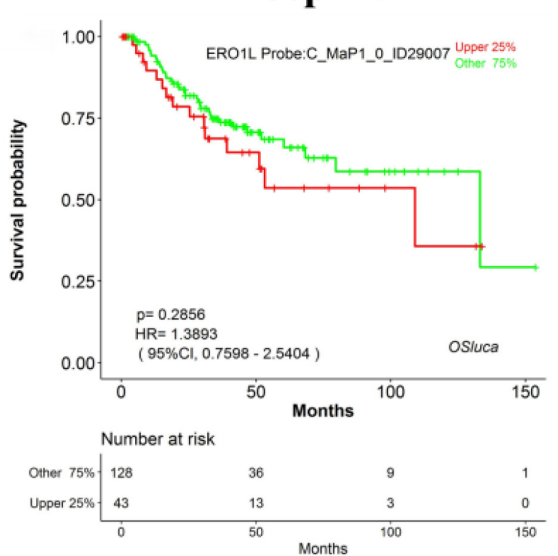

FIGURE 3 | Validation of a previously reported biomarker ERO1L in OSluca. Overexpression of ERO1L in tumor tissue is suggested as a worse survival biomarker in lung adenocarcinoma. (A) Overall survival (OS) of ERO1L gene in GSE30219 cohort. (B) OS in GSE31210 cohort. (C) OS in GSE37745 cohort. (D) OS in GSE41271 cohort. (E) OS in GSE50081 cohort. (F) OS in GSE67639 cohort. (G) OS in GSE68465 cohort. (H) OS in TCGA in lung adenocarcinoma. (I) OS in Roepman cohort. The histological type of all the above cohorts is lung adenocarcinoma. ERO1L, ERO1-like protein alpha (also named ERO1A).

servers of cancer (Elfilali et al., 2006; Mizuno et al., 2009; Goswami and Nakshatri, 2013; Gyõrffy et al., 2013; Tang et al., 2017) could substantially help researchers to discover potential biomarkers (Zheng et al., 2020). Herein, we developed a free web server OSluca to assess the prognostic value of the interesting gene in multiple cohorts of lung cancers. In OSluca, all the lung cancer cases are originated from the organ lung, not the second cancer from other cancers or 
organs. As a result, the prognostic specificity is only for lung cancer. Nevertheless, its prognostic significance in other types of cancers is also worth to be determined. To access the repeatability of previously reported prognostic biomarkers in OSluca, we collected 104 previously published prognostic biomarkers of lung cancer identified by $\mathrm{qPCR}$ or IHC, and tested their prognostic significance in OSluca. The testing results showed that most of the biomarkers were verified in OSluca and were confirmed for the published findings. Nevertheless, some genes showed different prognostic outcomes compared to previous literatures.

The advantage of OSluca over other online prognostic web servers is that the size of lung cancer samples in OSluca is large, and tens of independent cohorts are available, which is extremely valuable for the identification and validation of cancer prognostic biomarkers, since the most important part for the biomarker development is independent validation across different datasets/cohorts. The limitation of the current study is that OSluca can only test a single gene for outcome analysis. In summary, OSluca is a free web server for non-bioinformatics researchers to study potential lung cancer prognostic biomarkers, accessed at http://bioinfo.henu.edu.cn/LUCA/LUCAList.jsp.

\section{DATA AVAILABILITY STATEMENT}

The datasets generated for this study can be found in the TCGA, NCBI GEO, and Roepman dataset.

\section{REFERENCES}

Anaya, J. (2016). OncoLnc: linking TCGA survival data to mRNAs, miRNAs, and lncRNAs. Peerj Comput. Sci. 2:e67.

Baty, F., Facompre, M., Kaiser, S., Schumacher, M., Pless, M., Bubendorf, L., et al. (2010). Gene profiling of clinical routine biopsies and prediction of survival in non-small cell lung cancer. Am. J. Respir. Crit. Care Med. 181, 181-188. doi: 10.1164/rccm.200812-1807OC

Beer, D. G., Kardia, S. L., Huang, C. C., Giordano, T. J., Levin, A. M., Misek, D. E., et al. (2002). Gene-expression profiles predict survival of patients with lung adenocarcinoma. Nat. Med. 8, 816-824. doi: 10.1038/nm733

Bild, A. H., Yao, G., Chang, J. T., Wang, Q., Potti, A., Chasse, D., et al. (2006). Oncogenic pathway signatures in human cancers as a guide to targeted therapies. Nature 439, 353-357. doi: 10.1038/nature04296

Boelens, M. C., van den Berg, A., Fehrmann, R. S., Geerlings, M., de Jong, W. K., te Meerman, G. J., et al. (2009). Current smoking-specific gene expression signature in normal bronchial epithelium is enhanced in squamous cell lung cancer. J. Pathol. 218, 182-191. doi: 10.1002/path.2520

Botling, J., Edlund, K., Lohr, M., Hellwig, B., Holmberg, L., Lambe, M., et al. (2013). Biomarker discovery in Non-small cell lung cancer: integrating gene expression profiling, meta-analysis, and tissue microarray validation. Clin. Cancer Res. 19, 194-204. doi: 10.1158/1078-0432.ccr-12-1139

Bray, F., Ferlay, J., Soerjomataram, I., Siegel, R. L., Torre, L. A., and Jemal, A. (2018). Global cancer statistics 2018: GLOBOCAN estimates of incidence and mortality worldwide for 36 cancers in 185 countries. Cancer J. Clin. 68, 394-424. doi: 10.3322/caac. 21492

Byers, L. A., Diao, L., Wang, J., Saintigny, P., Girard, L., Peyton, M., et al. (2013). An epithelial-mesenchymal transition gene signature predicts resistance to EGFR and PI3K inhibitors and identifies Axl as a therapeutic target for overcoming EGFR inhibitor resistance. Clin. Cancer Res. 19, 279-290. doi: 10.1158/10780432.CCR-12-1558

\section{AUTHOR CONTRIBUTIONS}

XG: research design. QW and XG: establish OSluca web server. $\mathrm{ZY}, \mathrm{ZL}$, and XS: deal with RNA sequencing with clinical data of lung cancer. ZY, LX, XS, LZ, YL, and XG: draft of the manuscript. YD, XS, LZ, PS, YL, TX, and JM: collect previously reported biomarkers of lung cancer. ZY, LX, LZ, WZ, YZ, and XG: critical revision of the manuscript.

\section{FUNDING}

This study was supported by the following funding: The Kaifeng Science and Technology Major Project (18ZD008), the National Natural Science Foundation of China (Nos. 81602362 and 81801569), the Program for Science and Technology Development in Henan Province (Nos. 162102310391, 172102210187, and 192102310302), the Program for Young Key Teacher of Henan Province (2016GGJS-214), the supporting grants of Henan University (Nos. 2015YBZR048 and B2015151), and the Yellow River Scholar Program (No. H2016012).

\section{SUPPLEMENTARY MATERIAL}

The Supplementary Material for this article can be found online at: https://www.frontiersin.org/articles/10.3389/fgene. 2020.00420/full\#supplementary-material

Der, S. D., Sykes, J., Pintilie, M., Zhu, C.-Q., Strumpf, D., Liu, N., et al. (2014). Validation of a histology-independent prognostic gene signature for early-stage, non-small-cell lung cancer including stage IA patients. J. Thorac. Oncol. 9, 59-64. doi: 10.1097/JTO.0000000000000042

Dong, H., Wang, Q., Zhang, G., Li, N., Yang, M., An, Y., et al. (2020). OSdlbcl: an online consensus survival analysis web server based on gene expression profiles of diffuse large B-cell lymphoma. Cancer Med. 9, 1790-1797. doi: 10.1002/cam4. 2829

Elfilali, A., Lair, S., Verbeke, C., La Rosa, P., Radvanyi, F., and Barillot, E. (2006). ITTACA: a new database for integrated tumor transcriptome array and clinical data analysis. Nucleic Acids Res. 34, D613-D616. doi: 10.1093/nar/gkj022

Gao, L., Qiu, H., Liu, J., Ma, Y., Feng, J., Qian, L., et al. (2017). KLF15 promotes the proliferation and metastasis of lung adenocarcinoma cells and has potential as a cancer prognostic marker. Oncotarget 8, 109952-109961. doi: 10.18632/ oncotarget. 21972

Gentles, A. J., Bratman, S. V., Lee, L. J., Harris, J. P., Feng, W., Nair, R. V., et al. (2015). Integrating tumor and stromal gene expression signatures with clinical indices for survival stratification of early-stage Non-small cell lung cancer. J. Natl. Inst. 107:djv211. doi: 10.1093/jnci/djv211

Goswami, C. P., and Nakshatri, H. (2013). PROGgene: gene expression based survival analysis web application for multiple cancers. J. Clin. Bioinform. 3:22. doi: 10.1186/2043-9113-3-22

Gyõrffy, B., Surowiak, P., Budczies, J., and Lánczky, A. (2013). Online survival analysis software to assess the prognostic value of biomarkers using transcriptomic data in non-small-cell lung cancer. PLoS One 8:e82241. doi: 10.1371/journal.pone.0082241

Hammerman, P. S., Lawrence, M. S., Voet, D., Jing, R., Cibulskis, K., Sivachenko, A., et al. (2012). Comprehensive genomic characterization of squamous cell lung cancers. Nature 489, 519-525. doi: 10.1038/nature11404

Hoadley, K. A., Yau, C., Hinoue, T., Wolf, D. M., Lazar, A. J., Drill, E., et al. (2018). Cell-of-origin patterns dominate the molecular classification of 10,000 tumors 
from 33 Types of cancer. Cell 173, 291.e6-304.e6. doi: 10.1016/j.cell.2018.0 3.022

Hou, J., Aerts, J., den Hamer, B., van Ijcken, W., den Bakker, M., Riegman, P., et al. (2010). Gene expression-based classification of non-small cell lung carcinomas and survival prediction. PloS One 5:e10312. doi: 10.1371/journal.pone.0010312

Hsu, C.-H., Hsu, C.-W., Hsueh, C., Wang, C.-L., Wu, Y.-C., Wu, C.-C., et al. (2016). Identification and characterization of potential biomarkers by quantitative tissue proteomics of primary lung adenocarcinoma. Mol. Cell. Proteomics 15, 2396-2410. doi: 10.1074/mcp.M115.057026

Jones, M. H., Virtanen, C., Honjoh, D., Miyoshi, T., Satoh, Y., Okumura, S., et al. (2004). Two prognostically significant subtypes of high-grade lung neuroendocrine tumours independent of small-cell and large-cell neuroendocrine carcinomas identified by gene expression profiles. Lancet 363 , 775-781. doi: 10.1016/s0140-6736(04)15693-6

Kuner, R., Muley, T., Meister, M., Ruschhaupt, M., Buness, A., Xu, E. C., et al. (2009). Global gene expression analysis reveals specific patterns of cell junctions in non-small cell lung cancer subtypes. Lung Cancer 63, 32-38. doi: 10.1016/j. lungcan.2008.03.033

Larsen, J. E., Pavey, S. J., Bowman, R., Yang, I. A., Clarke, B. E., Colosimo, M. L., et al. (2007a). Gene expression of lung squamous cell carcinoma reflects mode of lymph node involvement. Eur. Respir. J. 30, 21-25. doi: 10.1183/09031936. 00161306

Larsen, J. E., Pavey, S. J., Passmore, L. H., Bowman, R., Clarke, B. E., Hayward, N. K., et al. (2007b). Expression profiling defines a recurrence signature in lung squamous cell carcinoma. Carcinogenesis 28, 760-766. doi: 10.1093/carcin/ bgl207

Larsen, J. E., Pavey, S. J., Passmore, L. H., Bowman, R. V., Hayward, N. K., and Fong, K. M. (2007c). Gene expression signature predicts recurrence in lung adenocarcinoma. Clin. Cancer Res. 13, 2946-2954. doi: 10.1158/1078-0432.ccr06-2525

Lee, E.-S., Son, D.-S., Kim, S.-H., Lee, J., Jo, J., Han, J., et al. (2008). Prediction of recurrence-free survival in postoperative Non-small cell lung cancer patients by using an integrated model of clinical information and gene expression. Clin. Cancer Res. 14, 7397-7404. doi: 10.1158/1078-0432.ccr-07-4937

Liu, J., Lichtenberg, T., Hoadley, K. A., Poisson, L. M., Lazar, A. J., Cherniack, A. D., et al. (2018). An integrated tcga pan-cancer clinical data resource to drive high-quality survival outcome analytics. Cell 173, 400.e11-416.e11. doi: 10.1016/j.cell.2018.02.052

Lu, Y., Lemon, W., Liu, P.-Y., Yi, Y., Morrison, C., Yang, P., et al. (2006). A gene expression signature predicts survival of patients with stage I non-small cell lung cancer. PLoS Med. 3:e030467. doi: 10.1371/journal.pmed.0030467

Mao, Y., Yang, D., He, J., and Krasna, M. J. (2016). Epidemiology of lung cancer. Surg. Oncol. Clin. North Am. 25, 439-445. doi: 10.1016/j.soc.2016.02.001

Meyerson, M., and Carbone, D. (2005). Genomic and proteomic profiling of lung cancers: lung cancer classification in the age of targeted therapy. J. Clin. Oncol. 23, 3219-3226. doi: 10.1200/JCO.2005.15.511

Mitchell, K. A., Zingone, A., Toulabi, L., Boeckelman, J., and Ryan, B. M. (2017). Comparative transcriptome profiling reveals coding and noncoding RNA differences in NSCLC from african americans and european americans. Clin. Res. 23, 7412-7425. doi: 10.1158/1078-0432.ccr-17-0527

Mizuno, H., Kitada, K., Nakai, K., and Sarai, A. (2009). PrognoScan: a new database for meta-analysis of the prognostic value of genes. BMC Med. Genomics 2:18. doi: $10.1186 / 1755-8794-2-18$

Okayama, H., Kohno, T., Ishii, Y., Shimada, Y., Shiraishi, K., Iwakawa, R., et al. (2012). Identification of genes up-regulated in ALK-positive and EGFR/KRAS/ALK-negative lung adenocarcinomas. Cancer Res. 72, 100-111. doi: 10.1158/0008-5472.can-11-1403

Ramalingam, S. S., Owonikoko, T. K., and Khuri, F. R. (2011). Lung cancer: new biological insights and recent therapeutic advances. Cancer J. Clin. 61, 91-112. doi: $10.3322 /$ caac. 20102

Raponi, M., Zhang, Y., Yu, J., Chen, G., Lee, G., Taylor, J. M., et al. (2006). Gene expression signatures for predicting prognosis of squamous cell and adenocarcinomas of the lung. Cancer Res. 66, 7466-7472. doi: 10.1158/00085472.can-06-1191

Robles, A. I., Arai, E., Mathé, E. A., Okayama, H., Schetter, A. J., Brown, D., et al. (2015). An integrated prognostic classifier for stage I lung adenocarcinoma based on mRNA, microRNA, and DNA methylation biomarkers. J. Thora.c Oncol. 10, 1037-1048. doi: 10.1097/JTO.0000000000000560
Roepman, P., Jassem, J., Smit, E. F., Muley, T., Niklinski, J., van de Velde, T., et al. (2009). An Immune response enriched 72-gene prognostic profile for earlystage non-small-cell lung cancer. Clin. Cancer Res. 15, 284-290. doi: 10.1158/ 1078-0432.ccr-08-1258

Rousseaux, S., Debernardi, A., Jacquiau, B., Vitte, A.-L., Vesin, A., Nagy-Mignotte, H., et al. (2013). Ectopic activation of germline and placental genes identifies aggressive metastasis-prone lung cancers. Sci. Transl. Med. 5:ra66. doi: 10.1126/ scitranslmed.3005723

Sato, M., Larsen, J. E., Lee, W., Sun, H., Shames, D. S., Dalvi, M. P., et al. (2013). Human lung epithelial cells progressed to malignancy through specific oncogenic manipulations. Mol. Cancer Res. 11, 638-650. doi: 10.1158/15417786.MCR-12-0634- $\mathrm{T}$

Shedden, K., Taylor, J. M. G., Enkemann, S. A., Tsao, M.-S., Yeatman, T. J., Gerald, W. L., et al. (2008). Gene expression-based survival prediction in lung adenocarcinoma: a multi-site, blinded validation study. Nat. Med. 14, 822-827. doi: $10.1038 / \mathrm{nm} .1790$

Staaf, J., Isaksson, S., Karlsson, A., Jonsson, M., Johansson, L., Jonsson, P., et al. (2013). Landscape of somatic allelic imbalances and copy number alterations in human lung carcinoma. Int. J. Cancer 132, 2020-2031. doi: 10.1002/ijc. 27879

Staaf, J., Jönsson, G., Jönsson, M., Karlsson, A., Isaksson, S., Salomonsson, A., et al. (2012). Relation between smoking history and gene expression profiles in lung adenocarcinomas. BMC Med. Genomics 5:22. doi: 10.1186/1755-8794-5-22

Takeuchi, T., Tomida, S., Yatabe, Y., Kosaka, T., Osada, H., Yanagisawa, K., et al. (2006). Expression profile-defined classification of lung adenocarcinoma shows close relationship with underlying major genetic changes and clinicopathologic behaviors. J. Clin. Oncol. 24, 1679-1688. doi: 10.1200/JCO.2005.03. 8224

Tang, Z., Li, C., Kang, B., Gao, G., Li, C., and Zhang, Z. (2017). GEPIA: a web server for cancer and normal gene expression profiling and interactive analyses. Nucleic Acids Res. 45, W98-W102. doi: 10.1093/nar/gkx247

The Cancer Genome Atlas Research Network (2012). Comprehensive genomic characterization of squamous cell lung cancers. Nature 489, 519525.

The Cancer Genome Atlas Research Network (2014). Comprehensive molecular profiling of lung adenocarcinoma. Nature 511, 543-550. doi: 10.1038/s41586018-0228-6

Tomida, S., Koshikawa, K., Yatabe, Y., Harano, T., Ogura, N., Mitsudomi, T., et al. (2004). Gene expression-based, individualized outcome prediction for surgically treated lung cancer patients. Oncogene 23, 5360-5370. doi: 10.1038/ sj.onc. 1207697

Tomida, S., Takeuchi, T., Shimada, Y., Arima, C., Matsuo, K., Mitsudomi, T., et al. (2009). Relapse-related molecular signature in lung adenocarcinomas identifies patients with dismal prognosis. J. Clin. Oncol. 27, 2793-2799. doi: 10.1200/JCO. 2008.19.7053

Wang, F., Wang, Q., Li, N., Ge, L., Yang, M., An, Y., et al. (2019a). OSuvm: an interactive online consensus survival tool for uveal melanoma prognosis analysis. Mol. Carcinog. 59, 56-61. doi: 10.1002/mc.23128

Wang, F., Wang, Q., Li, N., Ge, L., Yang, M., An, Y., et al. (2020). OSuvm: an interactive online consensus survival tool for uveal melanoma prognosis analysis. Mol. Carcinog. 59, 56-61.

Wang, Q., Xie, L., Dang, Y., Sun, X., Xie, T., Guo, J., et al. (2019b). OSlms: a web server to evaluate the prognostic value of genes in leiomyosarcoma. Front. Oncol. 9:190. doi: 10.3389/fonc.2019.00190

Wang, Q., Zhang, L., Yan, Z., and Xie, L. (2019c). OScc: an online survival analysis web server to evaluate the prognostic value of biomarkers in cervical cancer. Future Oncol. 15, 3693-3699. doi: 10.2217/fon-2019-0412

Wilkerson, M. D., Yin, X., Hoadley, K. A., Liu, Y., Hayward, M. C., Cabanski, C. R., et al. (2010). Lung squamous cell carcinoma mRNA expression subtypes are reproducible, clinically important, and correspond to normal cell types. Clin. Cancer Res. 16, 4864-4875. doi: 10.1158/1078-0432.CCR-10-0199

Wilkerson, M. D., Yin, X., Walter, V., Zhao, N., Cabanski, C. R., Hayward, M. C., et al. (2012). Differential pathogenesis of lung adenocarcinoma subtypes involving sequence mutations, copy number, chromosomal instability, and methylation. PloS One 7:e36530. doi: 10.1371/journal.pone.0036530

Xie, L., Dang, Y., Guo, J., Sun, X., Xie, T., Zhang, L., et al. (2019a). High KRT8 expression independently predicts poor prognosis for lung adenocarcinoma patients. Genes 10:36. doi: 10.3390/genes10010036 
Xie, L., Wang, Q., Dang, Y., Ge, L., Sun, X., Li, N., et al. (2019b). OSkirc: a web tool for identifying prognostic biomarkers in kidney renal clear cell carcinoma. Future Oncol. 15, 3103-3110. doi: 10.3892/ol.2019.10440

Xie, L., Wang, Q., Nan, F., Ge, L., and Dang, Y. (2019c). OSacc: gene expressionbased survival analysis web tool for adrenocortical carcinoma. Cancer Manag Res. 11, 9145-9152. doi: 10.2147/cmar.s215586

Xie, Y., Xiao, G., Coombes, K. R., Behrens, C., Solis, L. M., Raso, G., et al. (2011). Robust gene expression signature from formalin-fixed paraffin-embedded samples predicts prognosis of non-small-cell lung cancer patients. Clin. Cancer Res. 17, 5705-5714. doi: 10.1158/1078-0432.CCR-11-0196

Yan, Z., Wang, Q., Sun, X., Ban, B., Lu, Z., Dang, Y., et al. (2019). OSbrca: a web server for breast cancer prognostic biomarker investigation with massive data from tens of cohorts. Front. Oncol. 9:1349. doi: 10.3389/fonc.2019.01349

Zhang, G., Wang, Q., Yang, M., Yao, X., Qi, X., An, Y., et al. (2020). OSpaad: an online tool to perform survival analysis by integrating gene expression profiling and long-term follow-up data of 1319 pancreatic carcinoma patients. Mol. Carcinog. 59, 304-310. doi: 10.1002/mc.23154

Zhang, G., Wang, Q., Yang, M., Yuan, Q., Dang, Y., Sun, X., et al. (2019). OSblca: a web server for investigating prognostic biomarkers of bladder cancer patients. Front. Oncol. 9:466. doi: 10.2217/fon-2019-0296
Zheng, H., Zhang, G., Zhang, L., Wang, Q., Li, H., Han, Y., et al. (2020). Comprehensive review of web servers and bioinformatics tools for cancer prognosis analysis. Front. Oncol. 10:68. doi: 10.3389/fonc.2020. 00068

Zhu, C.-Q., Ding, K., Strumpf, D., Weir, B. A., Meyerson, M., Pennell, N., et al. (2010). Prognostic and predictive gene signature for adjuvant chemotherapy in resected non-small-cell lung cancer. J.Clin. Oncol. 28, 4417-4424. doi: 10.1200/ JCO.2009.26.4325

Conflict of Interest: The authors declare that the research was conducted in the absence of any commercial or financial relationships that could be construed as a potential conflict of interest.

Copyright (c) 2020 Yan, Wang, Lu, Sun, Song, Dang, Xie, Zhang, Li, Zhu, Xie, $M a$, Zhang and Guo. This is an open-access article distributed under the terms of the Creative Commons Attribution License (CC BY). The use, distribution or reproduction in other forums is permitted, provided the original author(s) and the copyright owner(s) are credited and that the original publication in this journal is cited, in accordance with accepted academic practice. No use, distribution or reproduction is permitted which does not comply with these terms. 